\title{
Lipid Peroxidation in Hepatic Fibrosis
}

\author{
Ichiro Shimizu, Noriko Shimamoto, \\ Katsumi Saiki, Mai Furujo and Keiko Osawa
}

Additional information is available at the end of the chapter

http://dx.doi.org/10.5772/46180

\section{Introduction}

Hepatic fibrosis is a complex dynamic process which is mediated by death of hepatocytes and activation of hepatic stellate cells (HSCs). Lipid peroxidation including the generation of reactive oxygen species (ROS), transforming growth factor- $\beta$, and tumor necrosis factor- $\alpha$ can be implicated as a cause of hepatic fibrosis.

Damage of any etiology, such as infection with hepatitis C virus (HCV) or hepatitis B virus (HBV), heavy alcohol intake, and iron overload, to hepatocytes can produce oxygen-derived free radicals and other ROS derived from lipid peroxidative processes. Persistent production of ROS constitutes a general feature of a sustained inflammatory response and liver injury, once antioxidant mechanisms have been depleted. The major source of ROS production in hepatocytes is NADH and NADPH oxidases localized in mitochondria (Figure 1). NADH and NADPH oxidases leak ROS as part of its operation. Kupffer cells (hepatic resident macrophages), infiltrating inflammatory cells such as macrophages and neutrophils, and HSCs also produce ROS in the injured liver.

\section{Oxidative stress in liver injury}

ROS include the free radicals superoxide $\left(\mathrm{O}_{2}^{-}\right)$and hydroxyl radical (HO-) and non-radicals such as hydrogen peroxide $\left(\mathrm{H}_{2} \mathrm{O}_{2}\right)$. A number of reactive nitrogen species including nitric oxide (NO) and peroxynitrite (ONOO-) are also ROS. Superoxide production is mediated mainly by $\mathrm{NADH}$ oxidase. Hydrogen peroxide is more stable and membrane permeable in comparison to other ROS. Thus, hydrogen peroxide plays an important role in the intracellular signaling under physiological conditions. With respect to pathological actions, ROS participate in the development of liver disease. In this regard, hydrogen peroxide is converted into the hydroxyl radical, a harmful and highly reactive ROS, in the presence of transition metals such as iron (Figure 1). The hydroxyl radical is able to induce not only lipid peroxidation in the structure of membrane phospholipids, which results in irreversible 
modifications of cell membrane structure and function (membrane injury), but DNA cleavage (DNA injury) as well. Such a chain of events due to increased ROS production exceeding cellular antioxidant defense systems are called oxidative stress, inducing cell death.

Malondialdehyde (MDA) and 4-hydroxynonenal (HNE) (Figure 1), end products of lipid peroxidation, are discharged from destroyed hepatocytes into the space of Disse (Figure 2). Cells are well equipped to neutralize the effects of ROS by virtue of a series of the antioxidant protective systems, including superoxide dismutase (SOD), glutathione peroxidase, glutathione (GSH), and thioredoxin. Upon oxidation, GSH forms glutathione disulfide (GSSG).

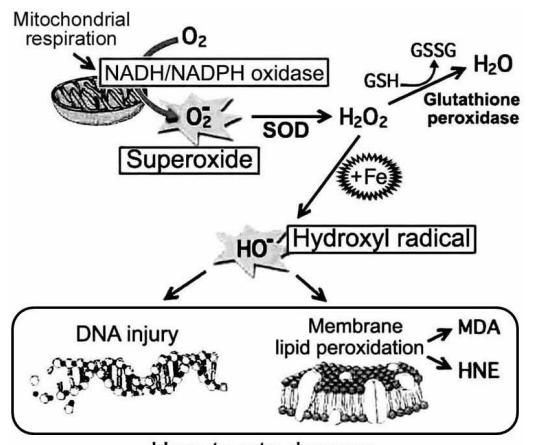

Hepatocyte damage

Figure 1. Oxidative stress and hepatocyte damage (Shimizu et al., 2012). A primary source of reactive oxygen species (ROS) production is mitochondrial NADPH/NADH oxidase. Hydrogen peroxide $\left(\mathrm{H}_{2} \mathrm{O}_{2}\right)$ is converted to a highly reactive ROS, the hydroxyl radical, in the presence of transition metals such as iron $(+\mathrm{Fe})$ and copper. The hydroxyl radical induces DNA cleavage and lipid peroxidation in the structure of membrane phospholipids, leading to cell death and discharge of products of lipid peroxidation, malondialdehyde (MDA) and 4-hydroxynonenal (HNE) into the space of Disse. Cells have comprehensive antioxidant protective systems, including SOD, glutathione peroxidase and glutathione (GSH). Upon oxidation, GSH forms glutathione disulfide (GSSG).

A single liver injury eventually results in an almost complete resolution, but the persistence of the original insult causes a prolonged activation of tissue repair mechanisms, thereby leading to hepatic fibrosis rather than to effective tissue repair. Hepatic fibrosis, or the excessive collagen deposition in the liver (see next section), is associated with oxidative stimuli and cell death. Cell death is a consequence of severe liver damage that occurs in many patients with chronic liver disease, regardless of the etiology such as $\mathrm{HCV} / \mathrm{HBV}$ infection, heavy alcohol intake, and iron overload.

\section{What is hepatic fibrosis?}

At the cellular levels, origin of hepatic fibrosis is initiated by the damage of hepatocytes, followed by the accumulation of neutrophils and macrophages including Kupffer cells on the sites of injury and inflammation in the liver. When hepatocytes are continuously damaged, leading to cell death, production of extracellular matrix proteins such as collagens 
predominates over hepatocellular regeneration. Overproduced collagens are deposited in injured areas instead of destroyed hepatocytes. In other words, hepatic fibrosis is fibrous scarring of the liver in which excessive collagens build up along with the duration and extent of persistence of liver injury. Hepatic fibrosis itself causes no symptoms but can lead to the end-stage cirrhosis. In cirrhosis the failure to properly replace destroyed hepatocytes and the excessive collagen deposition to distort blood flow through the liver (portal hypertension) result in severe liver dysfunction. Cirrhosis is an important host-related risk factor for the development of hepatocellular carcinoma (HCC) in chronic hepatitis C and B, as well as a major factor predicting a poor response to interferon-based antiviral therapy in chronic hepatitis C. Staging of chronic liver disease by assessment of hepatic fibrosis always is a major function of prognostic interpretation of individual data including liver biopsy. Of the commonly used staging systems, the METAVIR fibrosis score has been widely used (Huwart et al., 2008). The stages are determined by both the quantity and location of the fibrosis. With this score, F0 represents no fibrosis; F1 (mild fibrosis), portal fibrosis without septa; F2 (moderate fibrosis), portal fibrous and few septa; F3 (severe fibrosis), numerous septa without cirrhosis; and F4, cirrhosis (Figure 3), the tissue is eventually composed of nodules surrounded completely by fibrosis.

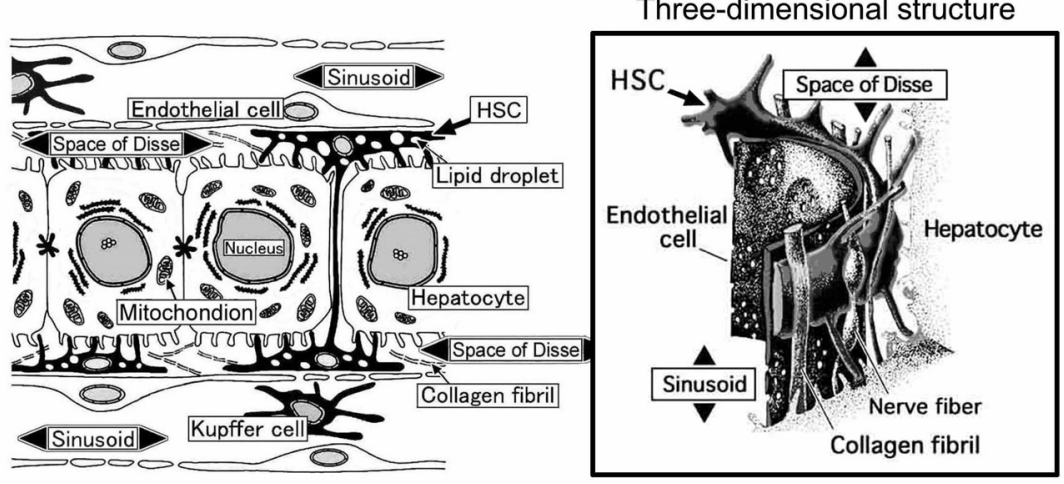

Figure 2. Schema of the sinusoidal wall of the liver (Shimizu et al., 2012). Schematic representation of hepatic stellate cells (HSCs) was based on the studies by Wake (Wake, 1999). Kupffer cells (hepatic resident macrophages) rest on fenestrated endothelial cells. HSCs are located in the space of Disse in close contact with endothelial cells and hepatocytes, functioning as the primary retinoid storage area. Collagen fibrils course through the space of Disse between endothelial cells and the cords of hepatocytes.

\section{Activation of HSCs}

Normal liver has a connective tissue matrix which includes collagen type IV (non-fibrillary), glycoproteins such as fibronectin and laminin, and proteoglycans such as heparan sulphate. These comprise the low density basement membrane in the space of Disse. Following liver injury there is a 3- to 8-fold increase in the extracellular matrix which is of a high density interstitial type, containing fibril-forming collagens (types 1 and III) as well as fibronectin, 
hyaluronic acid and proteoglycans. Collagen types 1 and III are major components of the extracellular matrix, which is principally produced by cells known as HSCs. HSCs are located in the space of Disse in close contact with hepatocytes and sinusoidal endothelial cells (Figure 2). Their three-dimensional structure consists of the cell body and several long and branching cytoplasmic processes (Wake, 1999). In the resting liver, HSCs have intracellular droplets containing retinoids. Retinoids refer to a group of chemical compound associated with vitamin A. HSCs contain approximately $50-80 \%$ of the whole body stores of retinoids (Blomhoff et al., 1990). In contrast, in the injured liver, HSCs are regarded as the primary target cells for inflammatory and oxidative stimuli, and they are proliferated, enlarged and transformed into myofibroblast-like cells. These HSCs are referred to as activated cells and are responsible for the overproduction of collagens during hepatic fibrosis to cirrhosis. This activation is accompanied by a loss of cellular retinoids, and the synthesis of $\alpha$-smooth muscle actin ( $\alpha$-SMA), and large quantities of the major components of the extracellular matrix including collagen types I, III, and IV, fibronectin, laminin and proteoglycans. $\alpha$-SMA is produced by activated HSCs (myofibroblast-like cells) but not by resting (quiescent) HSCs, thereby a marker of HSC activation. Moreover, activated HSCs produce ROS and transforming growth factor- $\beta$ (TGF- $\beta$ ) (Figure 4 ). TGF- $\beta$ is a major fibrogenic cytokine, regulating the production, degradation and accumulation of the extracellular matrix in hepatic fibrosis. TGF- $\beta$ expression correlates with the extent of hepatic fibrosis (Castilla et al., 1991). This cytokine induces its own expression in activated HSCs, thereby creating a self-perpetuating cycle of events, referred to as an autocrine loop. TGF- $\beta$ is also released in a paracrine manner from Kupffer cells, endothelial cells, and infiltrating inflammatory cells following liver injury. Similarly, ROS are produced by activated HSCs in response to ROS released from adjacent cells such as destroyed hepatocytes and activated Kupffer cells.

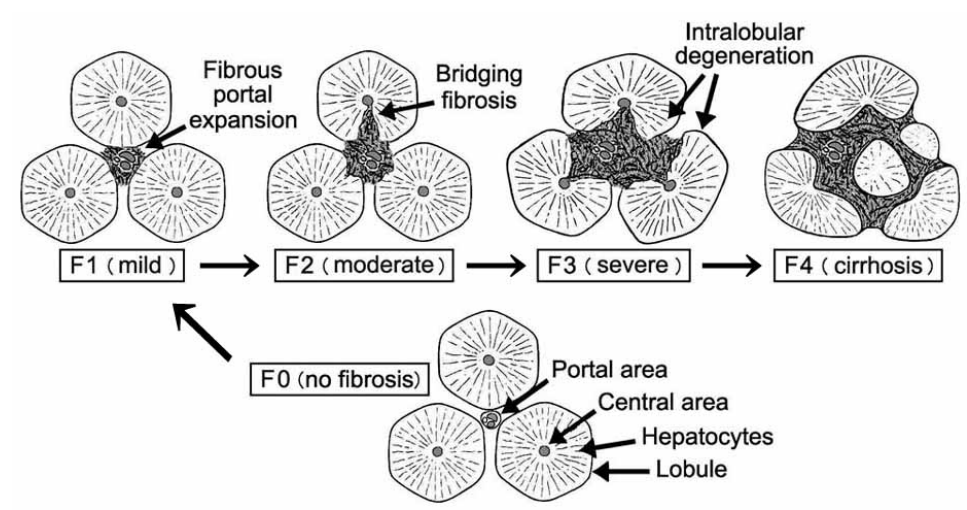

Figure 3. Stages of hepatic fibrosis in chronic hepatitis according to the five stages (0-4) of the METAVIR scoring system (1994). With this score, F0 represents no fibrosis; F1 (mild fibrosis), fibrous expansion of portal areas without septa; F2 (moderate fibrosis), fibrous septa extend to form bridges between adjacent vascular structures, both portal to portal and portal to central, occasional bridges; F3 (severe fibrosis), numerous bridges or septa without cirrhosis; and F4 (cirrhosis), the tissue is eventually composed of nodules surrounded completely by fibrosis. 


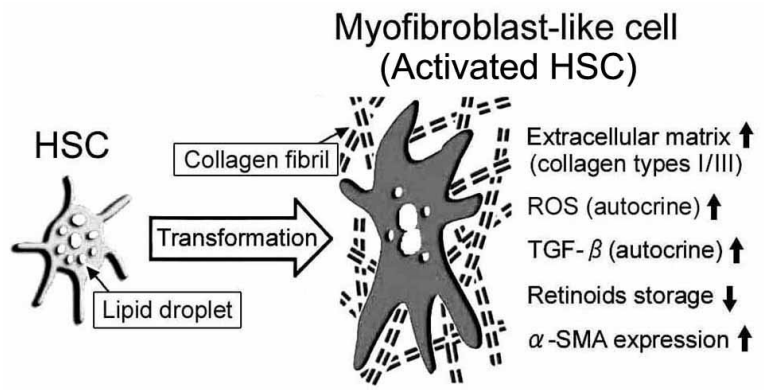

Figure 4. During liver injury, HSCs are proliferated, enlarged and transformed into myofibroblast-like cells (Shimizu, 2001). These activated HSCs produce large quantities of collagens, $\alpha$-smooth muscle actin ( $\alpha$-SMA), ROS, and transforming growth factor- $\beta$ (TGF- $\beta$ ), and lose cellular retinoids.

HSCs are activated mainly by ROS, products of lipid peroxidation (MDA and HNE) (Lee et al., 1995; Parola et al., 1993), and TGF- $\beta$, which are released from destroyed hepatocytes, activated Kupffer cells and infiltrating macrophages and neutrophils in the injured liver (Figure 5). In addition to ROS, exogenous TGF- $\beta$ increases the production of ROS, particularly hydrogen peroxide, by HSCs, whereas the addition of hydrogen peroxide induces ROS and TGF- $\beta$ production and secretion by HSCs (De Bleser et al., 1999). This socalled autocrine loop of ROS by HSCs is regarded as mechanism corresponding to the autocrine loop of TGF- $\beta$ which HSCs produce in response to this cytokine with an increased collagen expression in the injured liver (Itagaki et al., 2005).

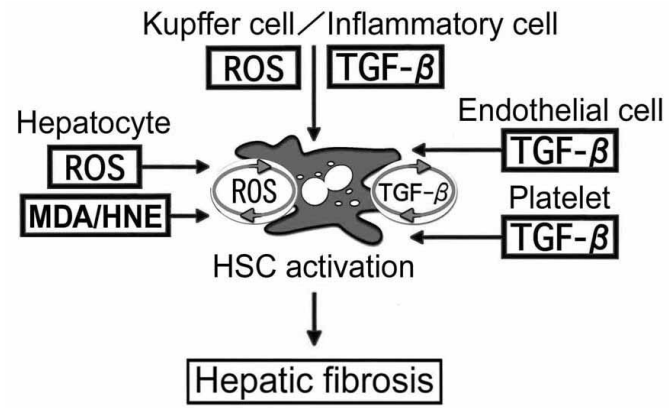

Figure 5. Activation of HSCs. HSCs are activated by such factors as ROS, lipid peroxidation products (MDA and HNE), and TGF- $\beta$ released when adjacent cells including hepatocyte, Kupffer cells, and endothelial cells are injured. ROS and TGF- $\beta$ are also produced by HSCs in response to exogenous ROS and TGF- $\beta$ in an autocrine manner.

Other important factors for HSC activation are platelet-derived growth factor (PDGF) released from platelets, and endothelin-1 from endothelial cells. PDGF is the most potent mitogen. HSCs congregate in the area of injury, through proliferation and migration from elsewhere, in response to the release of PDGF and monocyte chemotactic peptide-1 (MCP-1). MCP-1 is produced by activated Kupffer cells and infiltrating macrophages and neutrophils. The number of activated HSCs also increases after liver injury (Enzan et al., 1994). 
At the molecular levels, HSCs express the genes which encode for enzymes such as matrix metalloproteinase (MMP)-1 (interstitial collagenase) (Casini et al., 1994), which digests native fibrillar collagen types I and III, and MMP-2 (Milani et al., 1994), which digests denatured collagen types I and III and native collagen type IV, as well as tissue inhibitors of MMPs (TIMP)-1 and TIMP-2 (Iredale et al., 1992). Imbalance between matrix synthesis and degradation plays a major role in hepatic fibrosis (Shimizu, 2001). Matrix degradation depends upon the balance between MMPs, TIMPs and converting enzymes (MT1-MMP and stromelysin) (Li and Friedman, 1999). Collagen types I and III constitute the main framework of the so-called "fibrillar matrix". The space of Disse is a virtual space constituted by an extracellular matrix network composed of collagen type IV and noncollagenous components such as laminin. The large majority of collagen types III and IV, and laminin are synthesized by HSCs and endothelial cells, whereas all cell types synthesize small amounts of collagen type I. During hepatic fibrosis, however, HSCs become the major extracellular matrix producing cell type, with a predominant production of collagen type I (Maher and McGuire, 1990). In the resting liver, a balance between matrix synthesis and degradation exists, whereas, in the injured liver, the balance is disrupted. The net result of the changes during hepatocyte damage is increased degradation of the normal basement membrane collagen, and reduced degradation of interstitial-type collagens. The latter can be explained by increased TIMP-1 and TIMP-2 expressions relative to MMP-1. The degradative portion of the remodeling process is coordinated by MMPs and TIMPs.

\section{Oxidative stress and intracellular pathway}

Origin of hepatic fibrosis is initiated by the damage of hepatocytes, resulting in the recruitment of inflammatory cells and platelets, and activation of kupffer cells, with subsequent release of cytokines and growth factors. HSCs are the primary target cells for these inflammatory and oxidative stimuli, because during hepatic fibrosis, HSCs undergo an activation process to a myofibroblast-like cell, which represents the major matrix-producing cell. In the injured liver, hydrogen peroxide seems to act as a second messenger to regulate signaling events including mitogen activated protein kinase (MAPK) activation. The MAPK family includes three major subgroups, extracellular signal regulated kinase (ERK), p38 MAPK (p38), and c-Jun N-terminal kinase/stress activated protein kinase (JNK). MAPK participates in the intracellular signaling to: (1) induce the gene expression of redox sensitive transcription factors, such as activator protein-1 (AP-1) and nuclear factor $\kappa \mathrm{B}$ (NFкB) (Pinkus et al., 1996), (2) stimulate apoptosis (Clement and Pervaiz, 1999), and (3) modulate cell proliferation (Lundberg et al., 2000). ERK and JNK lie upstream of AP-1. JNK and p38 activation are more important in stress responses such as inflammation, which can also activate NF- $\kappa \mathrm{B}$. AP-1 and NF- $\kappa \mathrm{B}$ induce the expression of multiple genes involved in inflammation and oxidative stress response, cell death and fibrosis, including proinflammatory cytokines such as tumor necrosis factor- $\alpha$ (TNF- $\alpha$ ), interleukin-1 and interleukin- 6 and growth factors such as PDGF and TGF- $\beta$. TGF- $\beta$ is a major fibrogenic cytokine, acting as a paracrine and autocrine (from HSCs) mediator as already noted. TGF- $\beta$ 
triggers and activates the proliferation, enlargement and transformation of HSCs, but it exerts its inhibitory effect on hepatocyte proliferation (Nakamura et al., 1985).

Since many cytokines exert growth factor like activity, in addition to their specific proinflammatory effects, the distinction between cytokines and growth factors is somewhat artificial. No growth factor or cytokine acts independently. The injured liver, predominantly Kupffer cells and infiltrating macrophages and neutrophils, produces TNF- $\alpha$, interleukin-1 and interleukin-6. These proinflammatory cytokines may also also inhibit hepatic regeneration. In particular, TNF- $\alpha$ plays a dichotomous role in the liver, where it not only induces hepatocyte proliferation and liver regeneration but also acts as a mediator of cell death (Schwabe and Brenner, 2006). During TNF- $\alpha$-induced apoptosis in hepatocytes, hydrogen peroxide is an important mediator of cell death (Bohler et al., 2000).

In liver injury of hepatitis virus infection, transgenic mice expressing HBsAg exhibit the generation of oxidative stress and DNA damage, leading to the progression of hepatic fibrosis and carcinogenesis (Hagen et al., 1994; Nakamoto et al., 2004). In addition, HBV X protein changes the mitochondrial transmembrane potential and increases ROS production in the liver (Waris et al., 2001). Moreover, structural and non-structural (NS) proteins of HCV are involved in the production of ROS in an infected liver. HCV core protein is associated with increased ROS, decreased intracellular and/or mitochondrial glutathione content, and increased levels of lipid peroxidation products (Moriya et al., 2001). Glutathione is an antioxidant. NS3 protein of HCV activates NADPH oxidase in Kupffer cells to increase production of ROS, which can exert oxidative stress on nearby cells (Thoren et al., 2004).

\section{Conclusion}

Hepatic fibrosis is a complex dynamic process which is mediated by death of hepatocytes and activation of HSCs. Lipid peroxidation including the generation of ROS, TGF- $\beta$, and TNF- $\alpha$ can be implicated as a cause of hepatic fibrosis. HSCs are regarded as the primary target cells for inflammatory stimuli, and produce extracellular matrix components. HSCs are activated by such factors as ROS, lipid peroxidation products (MDA and HNE), and TGF- $\beta$ released when adjacent cells including hepatocyte, Kupffer cells, and endothelial cells are injured. ROS and TGF- $\beta$ are also produced by HSCs in response to exogenous ROS and TGF- $\beta$ in an autocrine manner. During TNF- $\alpha$-induced death in hepatocytes, ROS is an important mediator of cell death. The most common cause of hepatic fibrosis is currently chronic HCV/HBV infection.

Understanding the basic mechanisms underlying the ROS-mediated fibrogenesis provides valuable information on the search for effective antifibrogenic therapies.

\section{Author details}

Ichiro Shimizu*, Noriko Shimamoto, Katsumi Saiki, Mai Furujo and Keiko Osawa Showa Clinic, Shin Yokohama, Kohoku-ku, Yokohama, Kanagawa, Japan

* Corresponding Author 


\section{References}

The French METAVIR Cooperative Study Group (1994). Intraobserver and interobserver variations in liver biopsy interpretation in patients with chronic hepatitis C. Hepatology 20:15-20.

Blomhoff R, Green MH, Berg T \& Norum KR . (1990). Transport and storage of vitamin A. Science 250:399-404.

Bohler T, Waiser J, Hepburn H, Gaedeke J, Lehmann C, Hambach P, Budde K \& Neumayer $\mathrm{HH}$. (2000). TNF-alpha and IL-1alpha induce apoptosis in subconfluent rat mesangial cells. Evidence for the involvement of hydrogen peroxide and lipid peroxidation as second messengers. Cytokine 12:986-991.

Casini A, Ceni E, Salzano R, Milani S, Schuppan D \& Surrenti C . (1994). Acetaldehyde regulates the gene expression of matrix-metalloproteinase- 1 and -2 in human fatstoring cells. Life Sci 55:1311-1316.

Castilla A, Prieto J \& Fausto N . (1991). Transforming growth factors beta 1 and alpha in chronic liver disease. Effects of interferon alfa therapy. N Engl J Med 324:933-940.

Clement MV \& Pervaiz S . (1999). Reactive oxygen intermediates regulate cellular response to apoptotic stimuli: an hypothesis. Free Radic Res 30:247-252.

De Bleser PJ, Xu G, Rombouts K, Rogiers V \& Geerts A . (1999). Glutathione levels discriminate between oxidative stress and transforming growth factor-beta signaling in activated rat hepatic stellate cells. J Biol Chem 274:33881-33887.

Enzan H, Himeno H, Iwamura S, Saibara T, Onishi S, Yamamoto Y \& Hara H . (1994). Immunohistochemical identification of Ito cells and their myofibroblastic transformation in adult human liver. Virchows Arch 424:249-256.

Hagen TM, Huang S, Curnutte J, Fowler P, Martinez V, Wehr CM, Ames BN \& Chisari FV . (1994). Extensive oxidative DNA damage in hepatocytes of transgenic mice with chronic active hepatitis destined to develop hepatocellular carcinoma. Proc Natl Acad Sci U S A 91:12808-12812.

Huwart L, Sempoux C, Vicaut E, Salameh N, Annet L, Danse E, Peeters F, ter Beek LC, Rahier J, Sinkus R, Horsmans Y \& Van Beers BE . (2008). Magnetic resonance elastography for the noninvasive staging of liver fibrosis. Gastroenterology 135:32-40.

Iredale JP, Murphy G, Hembry RM, Friedman SL \& Arthur MJ . (1992). Human hepatic lipocytes synthesize tissue inhibitor of metalloproteinases-1. Implications for regulation of matrix degradation in liver. J Clin Invest 90:282-287.

Itagaki T, Shimizu I, Cheng X, Yuan Y, Oshio A, Tamaki K, Fukuno H, Honda H, Okamura Y \& Ito S . (2005). Opposing effects of oestradiol and progesterone on intracellular pathways and activation processes in the oxidative stress induced activation of cultured rat hepatic stellate cells. Gut 54:1782-1789.

Lee KS, Buck M, Houglum K \& Chojkier M . (1995). Activation of hepatic stellate cells by TGF alpha and collagen type I is mediated by oxidative stress through c-myb expression. J Clin Invest 96:2461-2468. 
Li D \& Friedman SL . (1999). Liver fibrogenesis and the role of hepatic stellate cells: new insights and prospects for therapy. J Gastroenterol Hepatol 14:618-633.

Lundberg AS, Hahn WC, Gupta P \& Weinberg RA . (2000). Genes involved in senescence and immortalization. Curr Opin Cell Biol 12:705-709.

Maher JJ \& McGuire RF . (1990). Extracellular matrix gene expression increases preferentially in rat lipocytes and sinusoidal endothelial cells during hepatic fibrosis in vivo. J Clin Invest 86:1641-1648.

Milani S, Herbst H, Schuppan D, Grappone C, Pellegrini G, Pinzani M, Casini A, Calabro A, Ciancio G \& Stefanini F . (1994). Differential expression of matrix-metalloproteinase-1 and -2 genes in normal and fibrotic human liver. Am J Pathol 144:528-537.

Moriya K, Nakagawa K, Santa T, Shintani Y, Fujie H, Miyoshi H, Tsutsumi T, Miyazawa T, Ishibashi K, Horie T, Imai K, Todoroki T, Kimura S \& Koike K . (2001). Oxidative stress in the absence of inflammation in a mouse model for hepatitis $C$ virus-associated hepatocarcinogenesis. Cancer Res 61:4365-4370.

Nakamoto Y, Suda T, Momoi T \& Kaneko S . (2004). Different procarcinogenic potentials of lymphocyte subsets in a transgenic mouse model of chronic hepatitis B. Cancer Res 64:3326-3333.

Nakamura T, Tomita Y, Hirai R, Yamaoka K, Kaji K \& Ichihara A . (1985). Inhibitory effect of transforming growth factor-beta on DNA synthesis of adult rat hepatocytes in primary culture. Biochem Biophys Res Commun 133:1042-1050.

Parola M, Pinzani M, Casini A, Albano E, Poli G, Gentilini P \& Dianzani MU . (1993). Stimulation of lipid peroxidation or 4-hydroxynonenal treatment increases procollagen (I) gene expression in human liver fat-storing cells. Biochem Biophys Res Commun 194:1044-1050.

Pinkus R, Weiner LM \& Daniel V . (1996). Role of oxidants and antioxidants in the induction of AP-1, NF-kappaB, and glutathione S-transferase gene expression. J Biol Chem 271:13422-13429.

Schwabe RF \& Brenner DA . (2006). Mechanisms of Liver Injury. I. TNF-alpha-induced liver injury: role of IKK, JNK, and ROS pathways. Am J Physiol Gastrointest Liver Physiol 290:G583-G589.

Shimizu I . (2001). Antifibrogenic therapies in chronic HCV infection. Curr Drug Targets Infect Disord 1:227-240.

Shimizu I, Kamochi M, Yoshikawa H \& Nakayama Y. (2012). Gender difference in alcoholic liver disease. In: Shimizu I (ed) Trends in alcoholic liver disease research: Clinical and scientific aspects. InTech-Open Access Publisher, Rijeka, Croatia, pp 23-40.

Thoren F, Romero A, Lindh M, Dahlgren C \& Hellstrand K . (2004). A hepatitis C virusencoded, nonstructural protein (NS3) triggers dysfunction and apoptosis in lymphocytes: role of NADPH oxidase-derived oxygen radicals. J Leukoc Biol 76:11801186.

Wake K . (1999). Cell-cell organization and functions of 'sinusoids' in liver microcirculation system. J Electron Microsc 48:89-98. 
Waris G, Huh KW \& Siddiqui A . (2001). Mitochondrially associated hepatitis B virus X protein constitutively activates transcription factors STAT-3 and NF-kappa B via oxidative stress. Mol Cell Biol 21:7721-7730. 